\title{
FIGO Stage IVA Ovarian Cancer
}

National Cancer Institute

\section{Source}

National Cancer Institute. FIGO Stage IVA Ovarian Cancer. NCI Thesaurus. Code C128094.

Ovarian cancer with pleural effusion with positive cytology. (FIGO, 2014) 